\title{
Elastomer-based Touch Sensor: Visualization of Tactile Pressure Distribution
}

\author{
Wanlin $\mathrm{Li}^{1}$, Jelizaveta Konstantinova ${ }^{1}$, Akram Alomainy ${ }^{2}$ \\ and Kaspar Althoefer ${ }^{1}$ \\ ${ }^{1}$ Centre for Advanced Robotics @ Queen Mary (ARQ) \\ Queen Mary University of London, Mile End Road, London, E1 4NS, United Kingdom \\ wanlin.li@gmul.ac.uk, j.konstantinova@qmul.ac.uk, \\ k.althoefer@qmul.ac.uk \\ ${ }^{2}$ School of Electronic Engineering and Computer Science (EECS) \\ Queen Mary University of London, Mile End Road, London, E1 4NS, United Kingdom \\ a.alomainy@qmul.ac.uk
}

\begin{abstract}
This paper presents an elastomer-based tactile sensor that can sense the tactile information in the form of pressure distribution. Our proposed sensor uses a piece of coated elastomer with thin conical pins underneath as the touch medium. The elastomer consists of 91 pins arranged in a honeycomb pattern, each pin can be regarded as a tactile sensing element. They are spaced at $1.5 \mathrm{~mm}$ in $x$ and $y$ direction. Each tactile element transfers the applied pressure value into a circular image pattern which can be captured by a camera placed at the end of the sensor structure. The applied pressure over the sensing array can be computed by processing the area of each sensing element. MATLAB is used to process the received images relating the applied pressure to the activated pixels in each circular pattern of the tactile element, and further visualizing the pressure distribution on a reconstructed surface of the sensor. This paper presents the development principle and fabrication process of the proposed sensor. The experimental results have proven the viability of the sensing concept; the prototype sensor can effectively detect single-point touch caused by objects with different dimensions and multi-point touch interactions with a spacing of more than $2.5 \mathrm{~mm}$.
\end{abstract}

Keywords: Tactile sensor, Elastomer-based.

\section{Introduction}

Tactile sensor development in robotics research is becoming an important aspect since touch sensing acquires useful information about the physical interaction with the external environment [1]. The human tactile system is extremely powerful as it consists of extraordinarily high number of epidermis mechanoreceptors to detect the mechanical stimulus on the skin. Different kinds of cells are aligned at different depths in human skin, called Pacinian Corpuscle, Ruffini Corpuscle, Merkel Cells and Meissner's Corpuscle [2]; these are sensitive to different kinds of mechanical stimuli, such as highfrequency vibration, static force, low-frequency force and low-frequency vibration. The 
human hand is one of the most dexterous parts and the feeling of touch is an essential human sensation. Figure 1 shows the cut-away view of a fingertip showing the intermediate ridges whose static and dynamic displacement are detected through the skin's mechanoreceptors [3].

Many biologically-inspired devices are embedded in robotic sensing systems. Today's robotic sensing systems consist of different kinds of tactile sensors [4] including resistive sensors, capacitive sensors, piezoelectric sensors, organic fieldeffect transistors (OFETs) sensors and optical sensors. These sensors are designed to acquire signals allowing tactile perception similar to the human and assist the robots in manipulation tasks. Tactile sensors can continuously provide tactile information, for instance the direction and the magnitude of forces at all contact points and the information (shape, texture, softness etc.) of the objects they are interacting with.

Optical tactile sensors have attracted plenty of attention from researchers in the past few years [5]. Compared with electromagnetic sensing methods such as resistive-based or capacitive-based sensors that largely require the transmission of electrical signals, optical-based sensors use an optical sensor, such as a high-resolution camera, to capture the information and interpret the deformation of the sensing surface as tactile sensation with the aid of computer vision techniques. Most of the optical sensors use a physical medium which deforms according to the applied force and then indirectly infers the pressure or force information from the deformation.

An ideal optical tactile sensor is required to be a compliant mechanism with a large sensing area and high sensitivity, but with low power consumption [6]. With the rapid development of small cameras and image processing techniques, many optical tactile sensors have been proposed and manufactured. The TacTip [7] is a biologicallyinspired sensing and object manipulation device that is inspired by the deformation of the epidermal layers of the human skin. Papillae pins on the inside of the device's skin are tracked by a camera and their movement under external contact can be converted into pressure and shear force. The tactile force sensor in [8] is a fiber optic based tactile array sensor that can detect normal forces and object shapes via nine individual sensing elements made from electromagnetically isolated optical fibers. The optical three-axis tactile sensor in [9] is capable of sensing normal force and shearing force detected from the deformation and displacement of the contact areas of rubber-made conical feelers. GelSight [10] has a soft contact interface, and provides high resolution tactile images of contact geometry, as well as contact force and slip conditions.

This paper introduces an optical-based tactile sensing concept employing a $2 \mathrm{D}$ vision system for the acquisition of tactile information, see Figure 1. The sensor uses a reflective soft opaque elastomer with 91 thin conical pins at one end made of silicone to obtain the spatial pressure distribution when physically interacting with objects. The conical pins are placed in a 3D printed shell that maintains contact with a transparent acrylic plate. Pressure is detected from the integrated binary values of bright pixels calculated from the captured image of the contact areas of the 91 conical pins. We present the design and development process in Section 2. The experimental evaluation process is presented in Section 3. Conclusions are drawn in Section 4. 


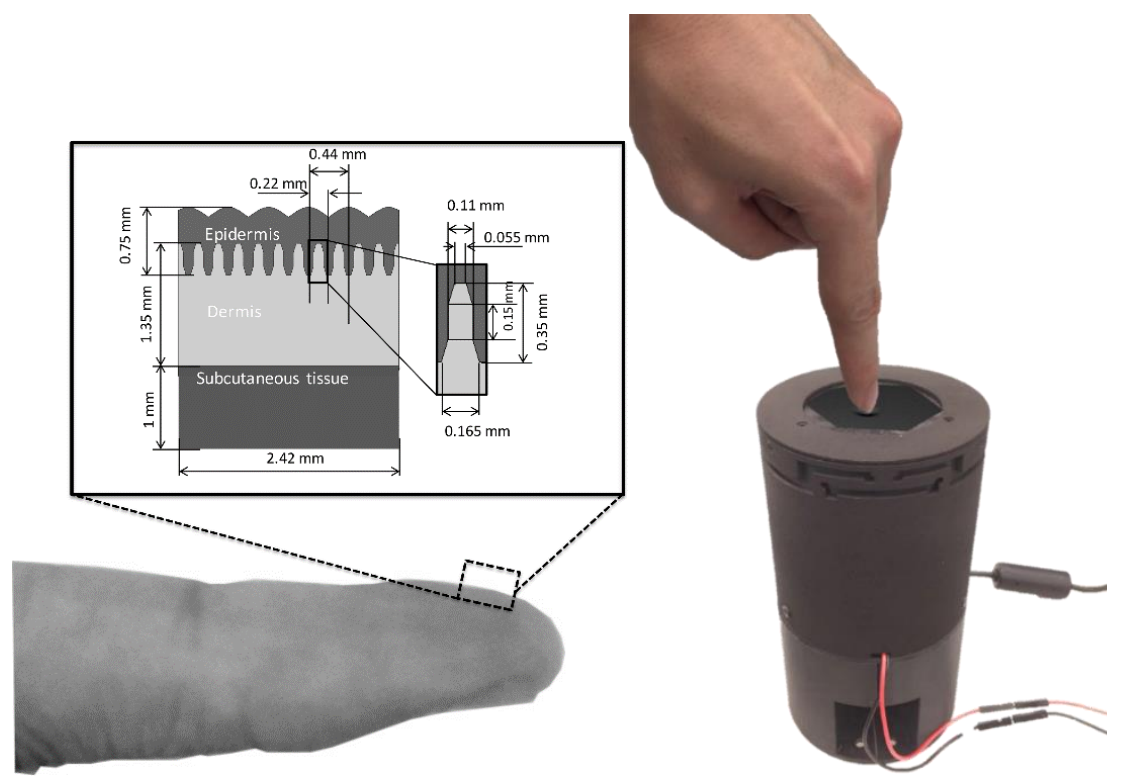

Fig. 1. A simplified elastic model mimicking a cut-away view of the human fingertip. The proposed tactile sensor can be used to create a 3D reconstruction of the sensor membrane. The Figure shown right is a sensory snapshot with its reconstruction shown together with the sensor.

\section{Sensor Design}

Our elastomer-based tactile sensor is a biologically-inspired and low-cost device which uses a 3D printed shell and a compliant contact surface (so-called elastomer) as a touch medium to obtain tactile information from stimuli. Figure 2 demonstrates the schematic design of the sensor that is made of several components. The elastomer (1) is made of a silicone material extracted from a 3D printed mold to provide the compliant properties referred as the tip of the sensor. The interior of the elastomer of an array of small reflective pins which are made of silicone mixed with metallic powder. More details of the elastomer will be shown in Section 2.1. A web-cam (2) is placed at the bottom of the device to track the pins of the elastomer. A transparent acrylic sheet with $5 \mathrm{~mm}$ thickness (3) is placed under the elastomer; this is the supporting plate to provide a solid plate for the camera to observe the deformation of the elastomer during contact. A white LED array (4) is placed inside the 3D printed shell (5) acting as an internal light source to illuminate the elastomer, the light goes through the light-guiding plate (6) which has a 45-degree angle at the edge towards the pins of the elastomer. The 3D printed sensor base (7) and shell are made of nylon (dyed in black color), the shell acts as a force sensitive structure (so-called flexure) behaving like a spring mechanism due to its build-in cantilever structure. With such a design, the flexure can support as well as stabilize the elastomer during the contact. A cap (8) is screwed onto the top of the sensor to keep the elastomer in place during the interaction with the external environment. 


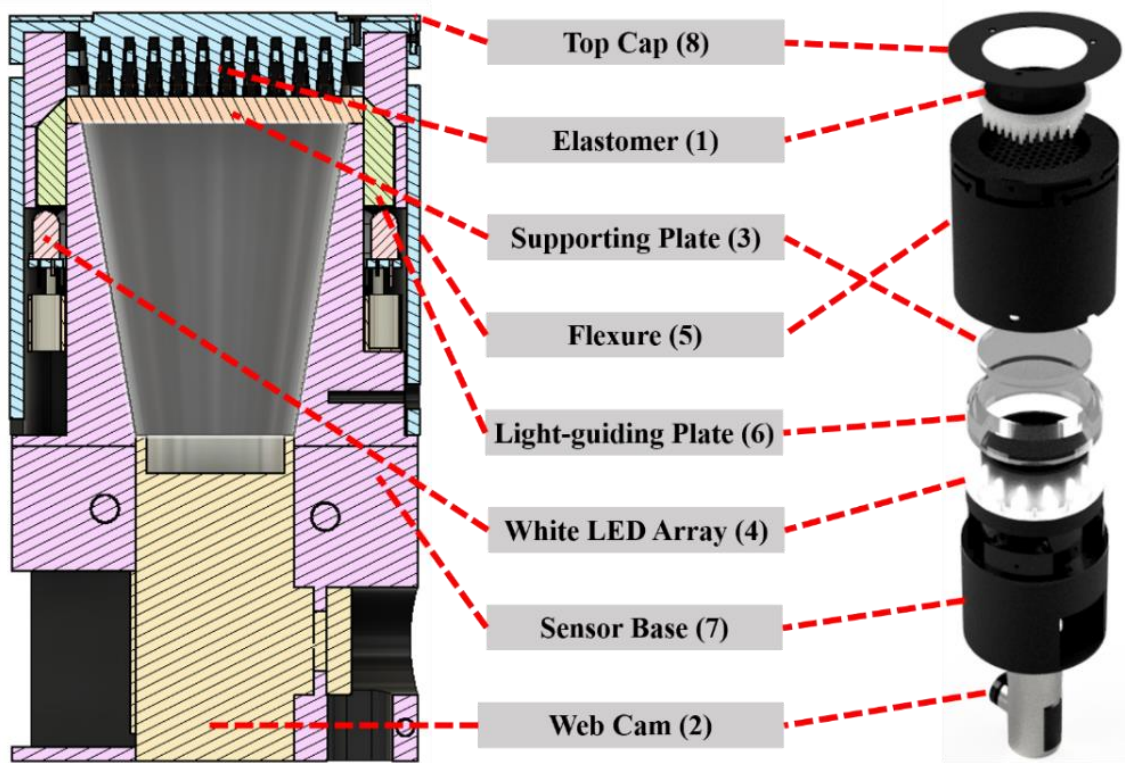

Fig. 2. Elastomer-based tactile sensor design. The left side shows the schematic design and the right side shows the exploded view of the sensor.

\subsection{Design and Fabrication of the Elastomer}

The elastomer part acts as the touch medium of the device and is composed of an array of pins on the interior of the soft silicone membrane to translate contact information into pin deformation, which can be then presented as the visualization of the tactile data. The array of pins performs a similar mechanical response as the dermal papillae of the human skin under load (see Figure 3).

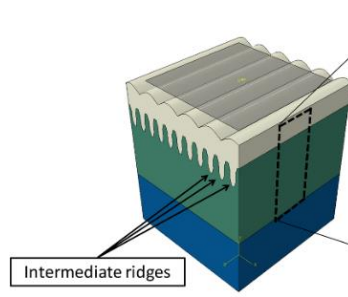

(a)

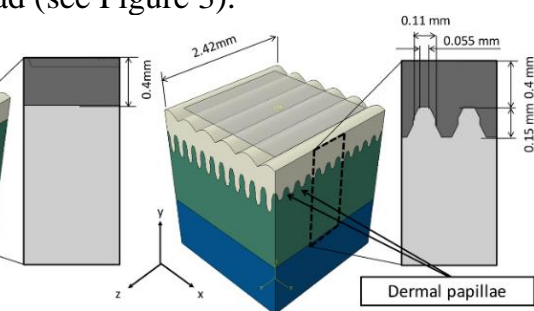

(b)

Fig. 3. Depth configuration of (a) the 2D ridge model and (b) the 3D ridge model.

In our proposed research, the elastomer part is composed of two parts: the opaque elastomer base with a hexagonal arrangement of 91 reflective pins underneath and the opaque membrane. The elastomer's properties largely influence the sensitivity of the device, so the pins need to be highly elastic and the membrane is made to be thin, flexible and uniform. Figure 4 (a) (b) (c) shows the manufactured elastomer including 
both elastomer base and the membrane together with its 3D printed mold. Our mold is made of the material Polylactide (PLA). The layout of the pins is a 2D hexagonal pattern with 91 elements which are $1.5 \mathrm{~mm}$ apart. Each conical pin has a height of 11 $\mathrm{mm}$, a diameter of $3 \mathrm{~mm}$ at the base and a diameter of $1.25 \mathrm{~mm}$ at its tip. These tactile pins are in contact with the transparent supporting plate, facing towards the camera when at rest and will be pushed towards the transparent plate when a load is exerted at the membrane on the top. This leads to a deformation of these conical pins captured by the camera at the base. The tactile information can then be extracted using computer vision methods.

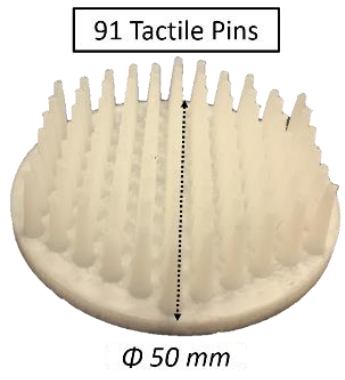

(a) Elastomer pins

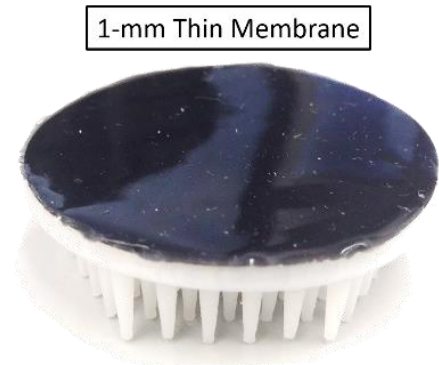

(b) Elastomer membrane

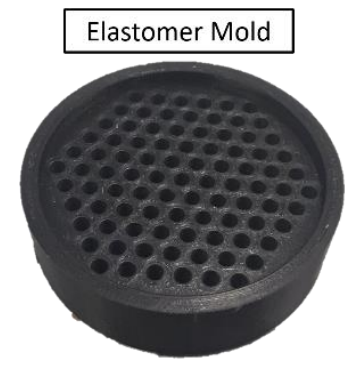

(c) Mold for elastomer

1)

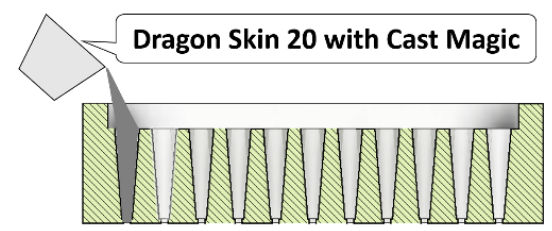

3)

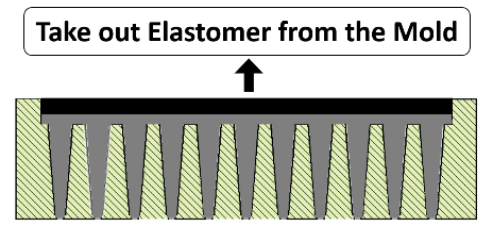

2)

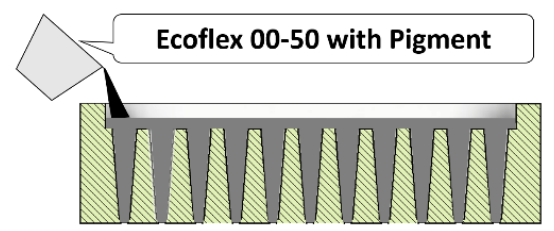

4)

(d) Fabrication process of elastomer

Fig. 4. Elastomer description and the fabrication process, with (a) and (b) showing the integrated elastomer. In (c), the mold for manufacturing the elastomer is depicted. In (d), the step-by-step manufacturing process of the elastomer with different parts using different types of silicone materials is shown.

Figure 4 (d) demonstrates the fabrication process of the elastomer part. The two types of silicone materials used in our sensor are Dragon Skin 20 and Ecoflex 00-50 from Smooth-on Company. The properties of both silicone types are shown in Table 1. During the molding of the elastomer base with its integrated pins, Silicone Thinner and part A of Dragon Skin 20 are pre-mixed with a weight ratio of 1:5. Silicone Thinner is 
a non-reactive silicone fluid that can lower the viscosity of platinum cure silicone (Dragon Skin and Ecoflex, in this case), which offers the advantages of making the elastomer de-air faster, as well as letting the liquid mixture better flow over the intricate mold with its many tinny holes. Then part B of Dragon Skin 20 is added into the liquid mixture with the same weight of part A. Silver Bullet Cast Magic Powder is then added to the material with a weight ratio of 1:100 to make the material reflective on its surface. The liquid mixture is put into a conditioning planetary mixer (THINKY ARE-250) to mix and degas. We pour the material into the holes of the mold and about four or five hours later the silicone will be cured and solidified. For the membrane, the layer is required to be thin and opaque to ensure high resolution of the sensor. Opacity helps to exclude any external light interference. Here we use Silc Pig silicone color pigment to blacken the silicone structure. To make the membrane, same colorant Silc Pig black is added to the part A of Ecoflex 00-50 and mix well before adding part B. The liquid mixture of Ecoflex 00-50 is then sent to the mixer and poured on the top of the cured elastomer base that is made of Dragon Skin 20. After 3 hours curing, the manufactured elastomer can be taken out of the mold.

Table 1. Elastomer Material Properties

\begin{tabular}{lll}
\hline Property & Dragon Skin 20 & Ecoflex 00-50 \\
\hline Specific Gravity & $1.08 \mathrm{~g} / \mathrm{cc}$ & $1.07 \mathrm{~g} / \mathrm{cc}$ \\
Cure Time & 4 hours & 3 hours \\
Shore Hardness & $20 \mathrm{~A}$ & $00-50$ \\
Tensile Strength & $550 \mathrm{psi}$ & $315 \mathrm{psi}$ \\
$100 \%$ Modulus & $49 \mathrm{psi}$ & $12 \mathrm{psi}$ \\
Elongation @ Break & $620 \%$ & $980 \%$ \\
Mix Ratio By Weight & $1 \mathrm{~A}: 1 \mathrm{~B}$ & $1 \mathrm{~A}: 1 \mathrm{~B}$ \\
Color & Translucent & Translucent \\
Mixed Viscosity & $20000 \mathrm{cps}$ & $8000 \mathrm{cps}$ \\
\hline
\end{tabular}

\subsection{Illumination and Image Capture}

As shown in Figure 5 (a), we use an LED array which contains 12 white 5 mm LEDs with a 30-degrees viewing angle. All LEDs are soldered on a hollow-ring shaped printed circuit board (PCB) in parallel and the LED array is placed inside of the sensor to generate the inner light source powered by a USB TTL cable. In order to completely block the external light interference, an opaque silicone rubber ring made of Ecoflex 0050 painted with black is placed on top of the light-guiding plate. The size of the hollowed rubber ring has an inner diameter of $55 \mathrm{~mm}$ and an outer diameter of $66 \mathrm{~mm}$ with 45-degree angle at one end, which is the same as the light-guiding plate dimension. The light-guiding plate is a 3D printed hollowed cylinder using transparent material VeroClear. It has a 45-degree angle on top end to guide and reflect the light from LED to the transparent supporting plate (shown in Figure 2 (6)), thus the reflective elastomer pins can be illuminated. The top cap of the sensor also helps to block the light coming from upwards. We use a webcam (Microsoft LifeCam Studio) with a resolution of $640 \times 480$ pixels and $30 \mathrm{fps}$ to capture the visible area of the elastomer pins. Focusing 
on the center area of $430 \times 430$ pixels cropped from the received raw image, tactile information can be processed and visualized in the form of pressure distribution. More details are presented in Section 3.1.

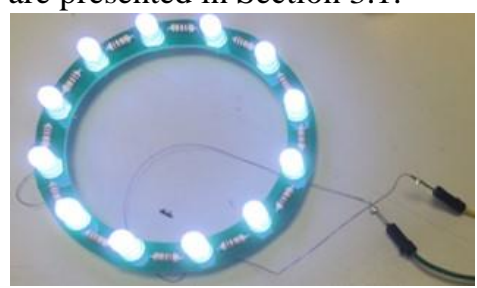

(a) Manufactured LED array

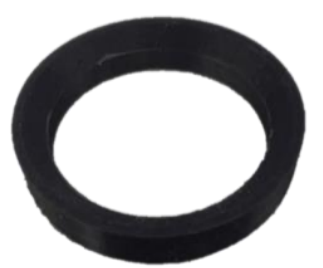

(b) Manufactured opaque rubber ring

Fig. 5. Proposed tactile sensor illumination system. In (a), the LED array provides the inner light source within a closed environment. In (b), an opaque rubber ring is placed within the flexure (Figure 2 (5)) to block the external light interference.

\section{Prototype Sensor Performance}

\subsection{Working Principle of Tactile Sensor}

A Matlab model determines the activated area of each tactile sensing element. As is shown in the flowchart of Figure 6, we firstly read the real-time video information from the web cam. Next, the real-time RGB image is converted to grayscale image and is divided into 91 hexagonal parts based on a uniformly distributed honeycomb layout which enables the detection of activated area for each sensing element. After that the grayscale image is further converted to binary image and a numeric display calculates the quantity of black and white pixels detected from each hexagonal area. Finally, via an interpolation method with the scattered data, we reconstruct a surface of the elastomer membrane based on the activated area of each silicone pin.

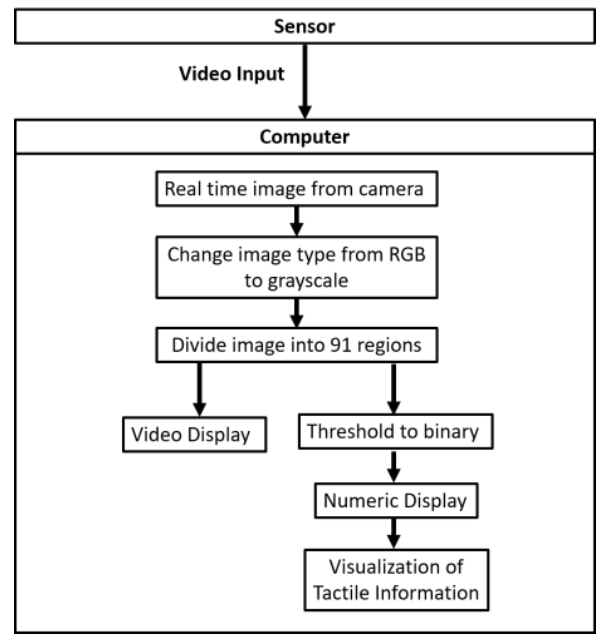

Fig. 6. Flowchart of activated pin areas to tactile information visualization system. The system acquires the input video in 30 frame per second and all the image computations can be processed in real-time. 
When switching on the power, the light is transferred from the source to the unloaded elastomer through the light-guiding plate and the supporting plate. The web cam is placed at the bottom to capture the raw images of the illuminated elastomer. The received images (without load) are displayed by the video output shown in Figure 7 (a), there are 91 pins on one side of the elastomer which are represented as the bright blobs. Then the input image is converted into a grayscale image and divided into 91 hexagonal parts (with a radius of 23 pixels each), see Figure 7 (b). Each hexagon represents one sensing element and all 91 elements can be treated as a tactile array. After thresholding, the grayscale image is converted into a binary image as shown in Figure 7 (c), there is no white blobs when no load is applied as the threshold eliminates the gray color. Once an external contact is applied on the elastomer membrane, the pins are squeezed onto the supporting plate, causing an increase in contact area with the supporting plate for each sensing element. These contact areas are white because of the reflectiveness of the pins and they can pass the threshold and generate the white blobs. Thus, the corresponding sensing area will generate more activated pixels. Figure 7 (d) (e) (f) shows the raw image received by the camera and the corresponding grayscale image and binary image when part of the black membrane (Figure 4 (b)) is touched on its left part. Figure 7 (g) (h) (i) shows the raw image received by the camera and the corresponding grayscale image and binary image when the whole surface of the membrane is touched and pressed.

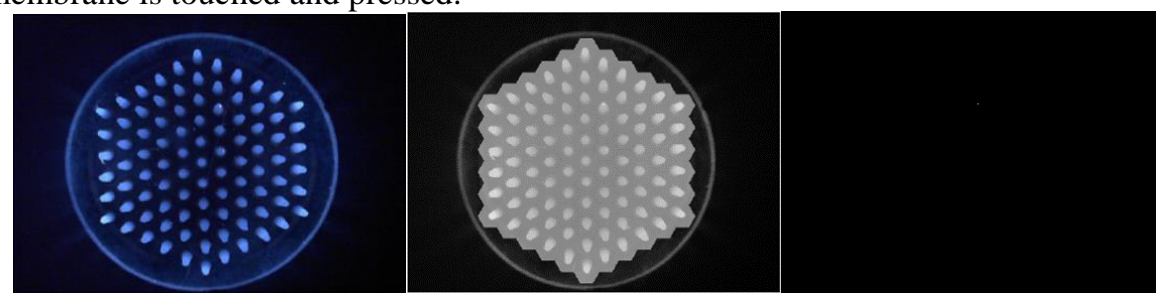

(a) raw image without load (b) grayscale without load (c) binary without load

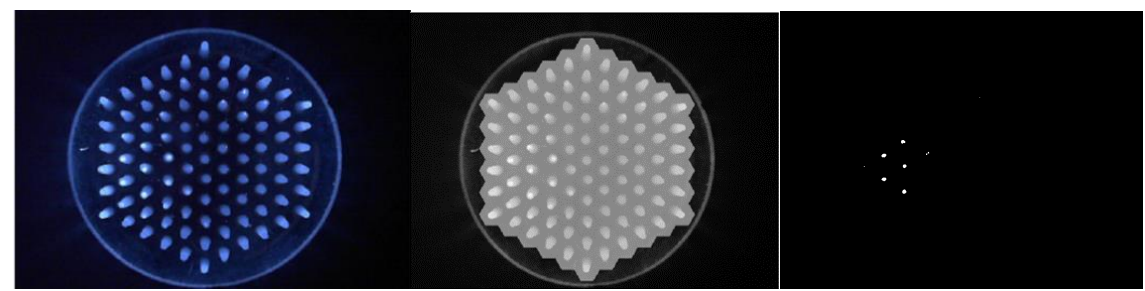

(d) Single-loaded raw image (e) Single-loaded grayscale (f) Single-loaded binary

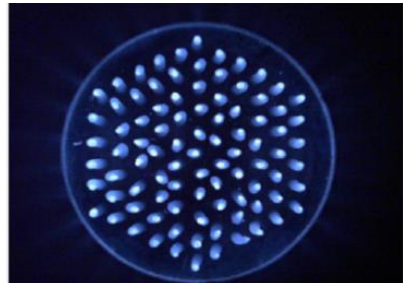

(g) Full-loaded raw image

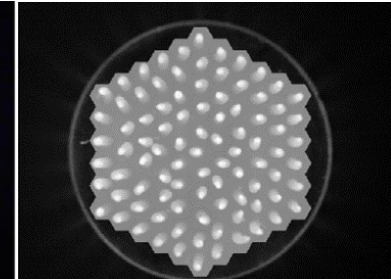

(h) Full-loaded grayscale

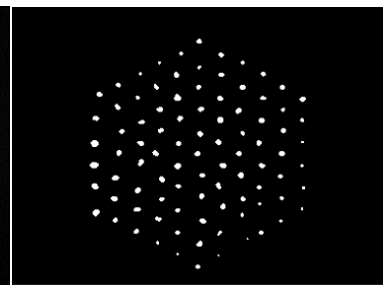

(i) Full-loaded binary

Fig. 7. Camera received image and viewer tool demonstrating the deformation of the reflective elastomer pins. 


\subsection{Visualization of Tactile Information}

The tactile information in terms of pressure distribution is measured in our proposed tactile sensor. The pressure distribution is visualized from the number of activated pixels of all 91 silicone pins. We use the scattered data interpolation method (griddata function in Matlab) to plot the interpolated surface of the elastomer membrane. We apply triangulation-based natural neighbor interpolation which is an efficient tradeoff between linear and cubic method to support 3-D interpolation. The grayscale pressure color map is built when an external contact is applied on the sensor membrane. When there is no contact, the initial reconstruction status of the surface in our method is shown in Figure 8 (a). To evaluate the tactile response of the proposed sensor, several tests including single-point touch, multiple-points touch are applied to the sensor. These tests aim to verify that the system can detect touch. Firstly, we test the single-point touch to the sensor as shown in Figure 8 (b). The sensor can detect and visualize the pressure on the reconstructed surface.
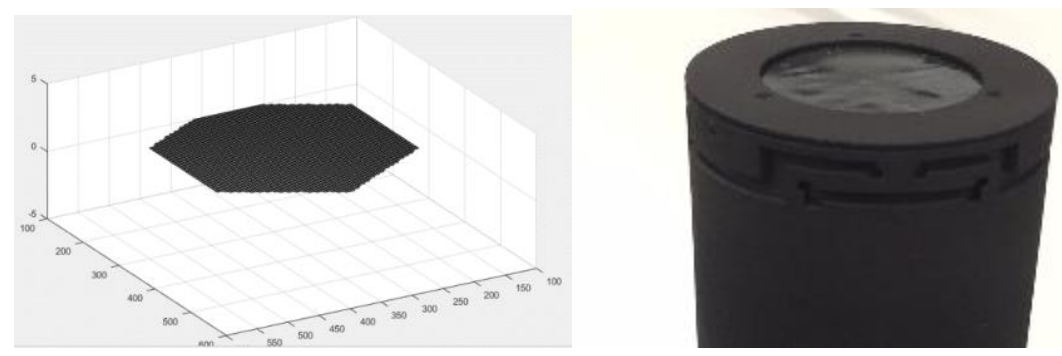

(a) No pressure is applied to sensor membrane

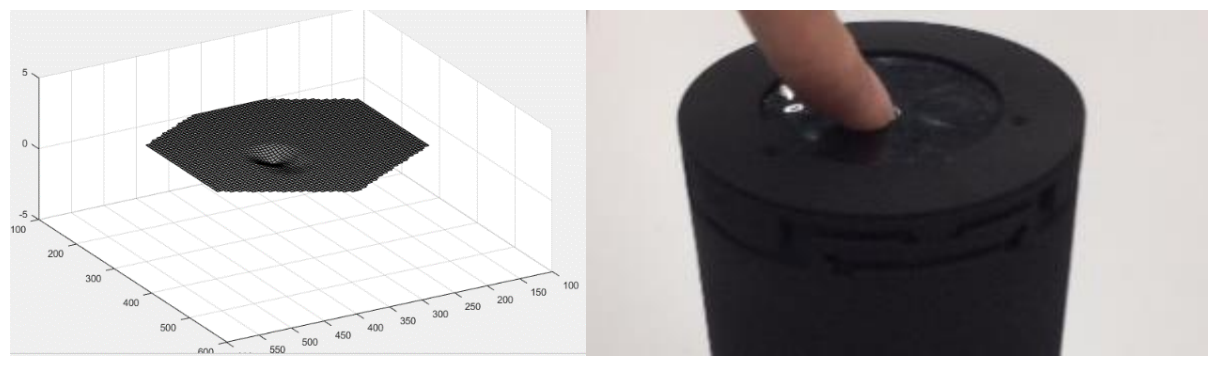

(b) Single-point contact to sensor membrane

Fig. 8. Reconstructing surface of the sensor membrane displaying the visualized tactile information in form of pressure distribution together with the corresponding pressure test.

Then we test the single-point touch with objects of different dimensions to investigate the touch ability (10 cubes with side length of $2 \mathrm{~mm}$ to $20 \mathrm{~mm}$ with an increment of $2 \mathrm{~mm}$, see Figure 9 (a)). We segment the sensor membrane into five zones as shown in Figure 9 (b), then we push each cube into each zone and calculate the sum of the activated pixels of the received images and repeat for 10 times. We then calculated the averaged activated pixels of the sensor for each cube under different zones and plot as shown in Figure 10. The results show that the sensor can detect 
different size of cubes over its whole surface and can recognize its corresponding dimensions.

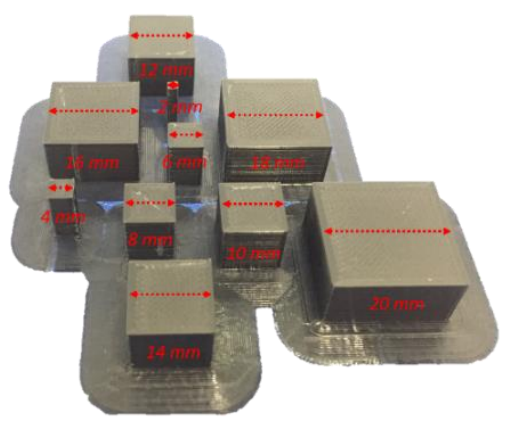

(a) 3D printed test cubes of different sizes

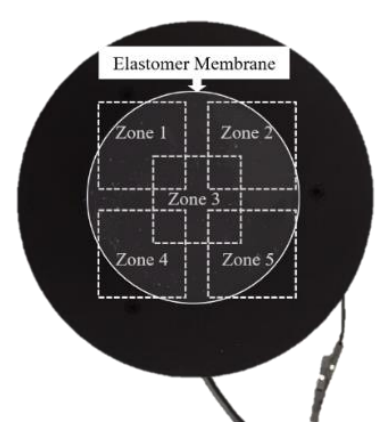

(b) 5 test zones of the membrane

Fig. 9. Setup for different pressure test. In (a), 10 different cubes are made to test the sensor touch ability. In (b), 10 cubes are placed one by one in Zone 1 to Zone 5 respectively and repeated 10 times for each test to record the sensor performance.
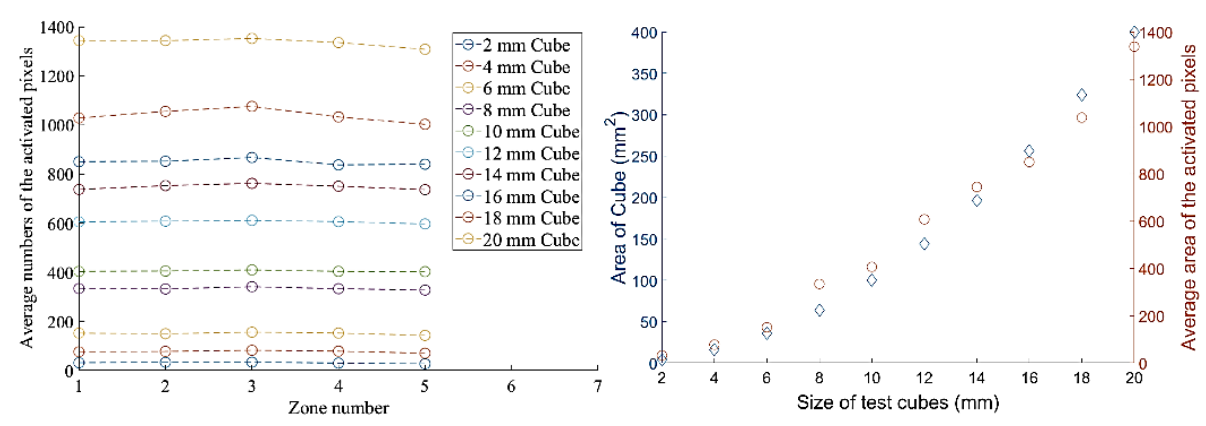

Fig. 10. The figure shown left is average number of the activated pixels while palpating cubes that differ in side length by $2 \mathrm{~mm}$. Each test repeats ten times for data collection. The figure shown right is the average number of the activated pixels of the sensor versus the areas of cubes.

Finally, we test with multi-points touch and the sensor is able to detect three-fingers touch as shown in Figure 11 (a). However, a misdetection happens when two fingers are very closed to each other as the sensor regards the touch as a single-point touch. The minimum spacing distance between multi-points is less than $2.5 \mathrm{~mm}$, as is shown in Figure 11 (b). 


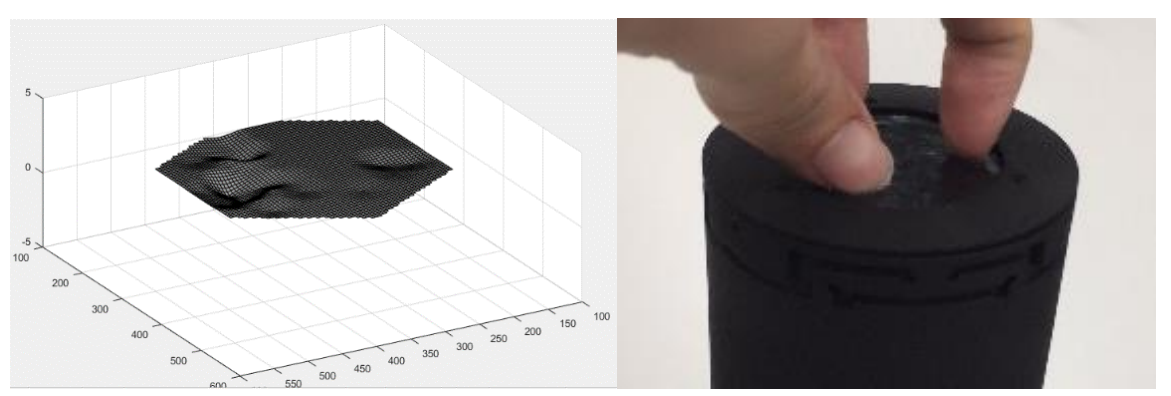

(a) Successful detection of multi-points contacts

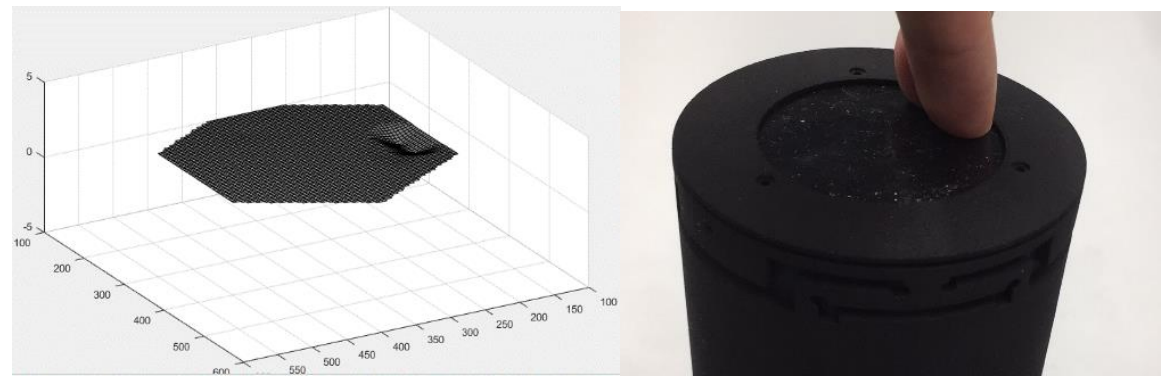

(b) Failed detection when contact points are less than $2.5 \mathrm{~mm}$ away from each other

Fig. 11. Reconstructing surface of the sensor membrane displaying the multi-points contact to the sensor. (a) shows a successful detect of three-point contact with human fingers. Failure happens in (b) when contact points are too closed to each other, specifically less than $2.5 \mathrm{~mm}$.

\section{Conclusions}

This paper presents a prototype tactile sensor that uses a soft coated elastomer with 91 thin conical pins at one end to sense the tactile information in the form of pressure distribution. The sensor can measure the tactile information over the sensor membrane surface based on a pixel-based method relying on the area change of each reflective conical pin recorded by a camera when a pressure is applied. The fabrication procedure shows the manufacturing of the elastomer using different silicone materials, as well as the other components of the sensor system. Test results show the feasibility of the sensing approach together with the accuracy of the sensor in detecting different objects. The proposed sensor has many advantages, e.g. compliant structure with the use of the elastomer, the sensor only uses one web cam to detect the sensing medium which provides a low-cost manufacturing. Future work will mainly focus on miniaturization of the sensor in order to fit with a wide range of robot arms and hands. An extension of the tactile spatial resolution may also be helpful to increase the sensing capability. 


\section{Acknowledgments}

This work was supported in part by the EPSRC National Centre for Nuclear Robotics project (EP/R02572X/1), the Innovate UK WormBot project (104059) and the Innovate UK project iGrasp (103676).

\section{References}

1. J. Konstantinova, A. Jiang, K. Althoefer, P. Dasgupta and T. Nanayakkara. Implementation of Tactile Sensing for Palpation in Robot-Assisted Minimally Invasive Surgery: A Review. In IEEE Sensors Journal, 14(8): 2490-2501, 2014.

2. R.S. Dahiya, G. Metta, M. Valle abd G. Sandini. Tactile sensing--from humans to humanoids. In IEEE Transactions on robotics, 26(1): 1-20, 2010.

3. T.Q. Pham, T. Hoshi, Y. Tanaka and A. Sano. Effect of 3D microstructure of dermal papillae on SED concentration at a mechanoreceptor location. In PloS one, 12(12): e0189293, 2017.

4. H. Yousef, M. Boukallel and K. Althoefer. Tactile sensing for dexterous in-hand manipulation in ro-botics-A review. In Sensors and Actuators A: physical, 167(2): 171187, 2011.

5. J.L. Schneiter and T.B. Sheridan. An optical tactile sensor for manipulators. Robot. Comput.-Integr. Manuf. 1984,

6. J. Konstantinova, A. Stilli and K. Althoefer. Fingertip Fiber Optical Tactile Array with TwoLevel Spring Structure. In Sensors 17(10), 2337, 2017.

7. B. Winstone, G. Griffiths, T. Pipe and J. Rossiter. TACTIP-Tactile fingertip device texture analysis through optical tracking of skin features. In Biomimetic Biohybrid Systems, pp. 323-334, 2013.

8. H. Xie, A. Jiang, H.A. Wurdemann and H. Liu. Magnetic resonance-compatible tactile force sensor using fiber optics and vision sensor. In IEEE Sensor Journal, 14(3): 829-838.

9. M. Ohka, Y. Mitsuya, I. Higashioka and H. Kabeshita. An experimental optical three-axis tactile sensor for micro-robots. In Robotica, 23(4): 457-465, 2005.

10. W. Yuan, R. Li, M.A. Srinivasan and E.H. Adelson. Measurement of shear and slip with a GelSight tactile sensor. In 2015 IEEE International Conference on Robotics and Automation (ICRA), pp. 304-311, 2015. 